\title{
Research on Identification of Petroliferous Unit Based on Seismic Data Structure Characteristics
}

\author{
Yu Ding ${ }^{1,2}$ Haimin Guo ${ }^{1,2}$ Moujie Li ${ }^{1,2, *}$ Changrong $\operatorname{Lin}^{3}$ and Xuyang Guo ${ }^{4}$
}

${ }^{1}$ Key Lab of Exploration Technologies for Oil and Gas Resources, Ministry of Education (Yangtze University), Wuhan 430100, China; ${ }^{2}$ School of Geophysics and Oil Resources, Yangtze University, Wuhan 430100, China; ${ }^{3}$ Production Institute of CNOOC Research Center, Beijing 101149, China; ${ }^{4}$ College of Geosciences, Texas A\&M University, the United States of America

\begin{abstract}
The identification and classification of petroliferous unit is the key to the success in the exploration for oil-gas fields with geophysical knowledge that are the main means and ways to reduce exploration risk and improve exploration efficiency. In this paper, it analyzes geophysical response characteristics under conditions of gas, oil and water through the establishment of horizontal layered medium model. Then it extracts and analyzes seismic abnormal characteristics of the data structure combining the method of grey system theory based on the experiment, which are considered as an important reference for further objectively identifying petroliferous unit and the exploration of oil-gas fields in the practical application with comparison and analysis.
\end{abstract}

Keywords: Data structure, geophysical knowledge, horizontal layered medium model, oil-gas fields, grey system theory.

\section{INTRODUCTION}

In the 1970s, the 'bright spot' technique had been used by geophysical workers for the identification of petroliferous unit with breakthrough. With the domanial expansion and technical development of oil-gas exploration, it is proposed AVO (Amplitude Versus Offset) technology in the systematic study on the basis of variational regularity with amplitude versus offsets that quickly gains widespread application. However, with the deepening and development of geophysical research work, it was found that variational regularity of oil-gas-water and the dipartition of petroliferous and nonpetroliferous formations could not be identified well alone with the change of amplitude characteristic in the case of oilgas formations, which mainly results from multiple solutions for seismic response characteristics. Under the guidance of seismic data structure theory put forward by Chinese famous scholar Prof. Lin Changrong, after taking a series of research, making use of seismic data structure characteristics is able to identify and classify petroliferous unit preferably that also can be considered as a basis for objectively understanding and solving oil-gas problems in practical application.

\section{BASIC PRINCIPLES FOR ANALYSIS OF SEISMIC DATA STRUCTURE}

The seismic data structure is the relationship of permutation and combination shown from discrete amplitude data of seismic wave [1], such as characteristic change in the slope and inflection point, etc. There are two aspects of content as follow:
1) permutation of chronological order in longitudinal direction; 2) relationship of space combination in transverse direction. To establish time-space relationship between the longitudinal and transverse is the premise for further study on petroliferous unit with distribution in formation space. This kind of data structure can not be identified only by simple geometric forms but using abnormal value of structure extracted from seismic data structure characteristics based on the GM $(1,1)$ model of the grey system theory [2]. To make an analysis of grey correlation degree between structural abnormal value and petroliferous characteristic value in order to achieve the purpose of identifying oil and gas, ultimately.

For the underground reservoir, it can be regarded as a grey system, differences between the lithology and fluid properties in it always show some kind of geophysical characteristics, which can be used to conduct seismic attribute analysis for predicting oil-gas possibility. However, there are different kinds of factors affecting the characteristic differences, each seismic attribute is only one of external manifestations of seismic data characteristics that exists a certain degree of flaws for the application in qualitative and semiquantitative analysis. Taken in this sense, the seismic data structure is an internal characteristic belonged to seismic data [3], compared in terms of seismic attributes, it is more stable and more suitable for quantitative and semiquantitative analysis.

Application of seismic data structure to achieve prediction for petroliferous property mainly includes two aspects of content: 1) to extract abnormal information of seismic data structure with the grey system theory in order to obtain abnormal data structure for the reflection of seismic data structure characteristics; 2) to determine abnormal response characteristics of seismic data structure based on correlation re- 
search between petroliferous property and seismic data structure abnormity using analysis of the grey correlation degree.

\subsection{Abnormal Information Extraction of Seismic Data Structure}

Differential equation model of the grey theory is known as the GM model, wherein, G stands for grey, $M$ stands for model. The GM $(1, \mathrm{~N})$ indicates first-order differential equation mode of the N-type variable. So, the $\operatorname{GM}(1,1)$ can be understood as first-order differential equation mode of a variable [4]. The method of using the $\operatorname{GM}(1,1)$ to predict quantity size for time sequence is known as the grey sequence prediction. The calculation steps of the GM $(1,1)$ modeling are as follow:

1) Parent sequence and sub-sequence

Given the original data sequence

$X^{(0)}=\left(X_{(1)}^{(0)}, X_{(2)}^{(0)}, \ldots, X_{(N)}^{(0)}\right)$,

where, the $\mathrm{N}$ is the number of data, the last data $X_{(N)}^{(0)}$ is called original point. If selecting continuous data (including original point) in different lengths from the $X^{(0)}$, it can form a new data sequence as follow:

$X_{(1)}^{(0)}=\left(X_{(2)}^{(0)}, X_{(3)}^{(0)}, \ldots, X_{(N)}^{(0)}\right)$

$X_{(2)}^{(0)}=\left(X_{(3)}^{(0)}, X_{(4)}^{(0)}, \ldots, X_{(N)}^{(0)}\right)$

$X_{(N-4)}^{(0)}=\left(X_{(N-3)}^{(0)}, X_{(N-2)}^{(0)}, \ldots, X_{(N)}^{(0)}\right)$.

In order to reduce the randomness of prediction sequence, the $\operatorname{GM}(1,1)$ adopts sequence length $\mathrm{N} \geq 4$ as general requirements.

2) Modeling of the GM $(1,1)$

(a) Selecting any sub-sequence denoted by

$X_{(i)}^{(0)}=\left(X_{(1)}^{(0)}, X_{(2)}^{(0)}, \ldots, X_{(N)}^{(0)}\right), \quad i=1,2, \ldots, N-3$.

(b) Making once accumulation generation for the subsequence,

$X^{(1)}=\left(X_{(1)}^{(1)}, X_{(2)}^{(1)}, \ldots, X_{(N)}^{(1)}\right)$.

Where, $X_{(1)}^{(1)}=X_{(1)}^{(0)}, X_{(t)}^{(1)}=\sum_{k=1}^{t} X_{K}^{(0)}, t=2,3, \ldots, N$.

Then, building the GM $(1,1)$ based on sequence $(2)$ :

$\frac{d X^{(1)}}{d t}+a X^{(1)}=u$.

^ Where, $a$ and $u$ are the model parameters denoted by $a=[a, u]^{T}$.

(c) Solving parameter $\hat{a}$ with the least squares method,

$\hat{a}=\left(B^{T} B\right)^{-1} B^{T} Y_{N}$

Where,
$B=\left[\begin{array}{cc}-\frac{1}{2}\left\{X_{(1)}^{(1)}+X_{(2)}^{(1)}\right\} & 1 \\ -\frac{1}{2}\left\{X_{(2)}^{(1)}+X_{(3)}^{(1)}\right\} & 1 \\ \cdots \cdots & \\ -\frac{1}{2}\left\{X_{(N-1)}^{(1)}+X_{(N)}^{(1)}\right\} & 1\end{array}\right]$,

$Y_{N}=\left\{X_{(2)}^{(0)}, X_{(3)}^{(0)}, \ldots, X_{(N)}^{(0)}\right\}^{T}, B^{T}$ is the transposed matrix of $B$.

(d) Substituting $\hat{a}$ into equation (3), the grey model can be written as

$\hat{X_{(t)}^{(1)}}=\left(X_{(1)}^{(1)}-\frac{u}{a}\right) e^{-a t}+\frac{u}{a}, \quad t=2,3, \ldots, N$.

(e) Making $\hat{X_{(t)}^{(1)}}$ derivation to the reduction of $\hat{X_{(t+1)}^{(0)}}$,

$\hat{X_{(t+1)}^{(0)}}=-a\left(X_{(1)}^{(0)}-\frac{u}{a}\right) e^{-a t}$.

Or, making $\hat{X_{(t)}^{(1)}}$ subtraction operation to the $\hat{X_{(t)}^{(0)}}$,

$\hat{X_{(t+1)}^{(0)}}=\hat{X_{(t+1)}^{(1)}}-\hat{X_{(t)}^{(1)}}$.

(f) Computing values of absolute error $e_{(t)}$ and relative error $q_{(t)}$ between $X_{(t)}^{(0)}$ and $X_{(t)}^{(0)}$,

$e_{(t)}=X_{(t)}^{(0)}-\hat{X_{(t)}^{(0)}}$

$q_{(t)}=\frac{e_{(t)}^{(0)}}{X_{(t)}^{(0)}}$.

\subsection{Grey Correlation Analysis}

Grey correlation analysis is a method for studying relevance between target sequence and comparison sequence. Generally speaking, there are one reference sequence $X_{0}$ and multiple comparison sequence $X_{i}$, which are applied to analyze and evaluate correlation degree between them. When the reservoir contains oil and gas, there will be shown a particular way of permutation and combination in seismic data known as the form of seismic data structure. In order to study petroliferous situation of subsurface reservoir, it is able to conduct correlation analysis between arbitrary seismic trace and the data structure with known petroliferous segment based on similarity of structural characteristics under that condition [5-6].

The specific calculation process of correlation analysis based on computation of correlation coefficient and degree is as follows:

Given that there are $\mathrm{N}+1$ data sequences and each one has M data,

$$
\begin{aligned}
& X_{0}(t)=\left(X_{0}(1), X_{0}(2), \ldots, X_{0}(M)\right) ; \\
& X_{1}(t)=\left(X_{1}(1), X_{1}(2), \ldots, X_{1}(M)\right) ;
\end{aligned}
$$

$$
X_{n}(t)=\left(X_{n}(1), X_{n}(2), \ldots, X_{n}(M)\right) \text {. }
$$


Generally, assuming $X_{0}(t)$ as the reference sequence (parent sequence) and $\left.X_{i}(t)\right|_{i=1,2, \ldots, n}$ as the comparison one (sub-sequence). Due to the inconsistent problems of dimension among different sequences, it usually takes dimensionless treatment with methods of initialization, equalization and normalization according to the actual situation so as to ensure the data obtain unified comparison standard [7].

Initialization refers to all the sample data are divided by the first sample value,

$X_{i}(j)=\frac{X_{i}(j)}{X_{i}(1)}$

Wherein, $X_{i}(j)$ is the value of the j-th data of the i-th data sequence, while $X_{i}(1)$ is the value of the first data of the ith data sequence.

Equalization refers to all the sample data are divided by sample average,

$X_{i}(j)=\frac{X_{i}(j)}{E\left(X_{i}(j)\right)}$.

Wherein, $E\left(X_{i}(j)\right)$ is the mathematical expectation of the $i$-th data sequence.

Normalization refers to all the sample data are divided by the maximum sample value,

$$
X_{i}(j)=\frac{X_{i}(j)}{\operatorname{MAX}\left(X_{i}(j)\right)} .
$$

Wherein, $\operatorname{MAX}\left(X_{i}(j)\right)$ is the maximum sample value of the i-th data sequence.

If taking $X_{0}$ as the reference sequence and $X_{i}, i=1,2, \ldots, n$ as the comparison one, the correlation coefficient of curves $X_{0}$ and $X_{i}$ at the k-th point can be represented by the following formula:

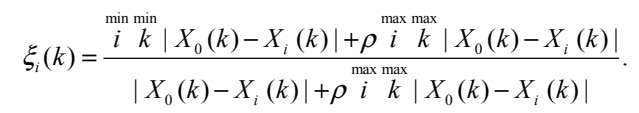

Wherein, $\xi_{i}(k)$ is known as correlation coefficient referred to the $\mathrm{k}$-th sample value of the $\mathrm{i}$-th sequence to the reference one; $\left|X_{0}(k)-X_{i}(k)\right|$ is called absolute difference referred to the $\mathrm{k}$-th sample value of the $\mathrm{i}$-th sequence to the k-thinthin sample value max of reference sequence; $i k\left|X_{0}(k)-X_{i}(k)\right|$ and $i k\left|X_{0}(k)-X_{i}(k)\right|$ is the minimum value and maximum value of absolute difference for all sequences with the reference sequence; $\rho$ is resolution coefficient and its value selection is 2 as often.

This can obtain correlation degree referred to sequences of comparison and reference through averaging for correlation coefficient to each sample value of comparison sequence.

$$
R_{i}=\frac{1}{N} \sum_{k=1}^{N} \varepsilon_{i}(k) .
$$

As a matter of fact, any seismic trace's petroliferous property is unknown and the change resulted from petroliferous differences in the data structure is also uncertain. Therefore, to conduct research for this uncertainty relationship in order to find relatively fixed objective laws under the conditions of uncertain factors and relationships that have research value with a certain degree. The greater advantage of the grey correlation analysis method is supposed to be well applied to the analysis and research.

\section{THINKING FOR PREDICTION OF PETROLIFER- OUS PROPERTY IN SEISMIC DATA STRUCTURE}

The reservoirs exist in underground traps with certain conditions, there must be some kind of external manifestations characteristics of natural law that show the features of geology and geophysics and geochemical effect in the earth's surface [8]. To analyze and identify these geophysical characteristics associated with oil and gas is considered as the process of identification and prediction for petroliferous property of reservoirs in seism.

As shown in Fig. (1), first of all, it establishes onedimensional prediction model GM $(1,1)$ by extracting amplitude data of seismic trace so as to obtain prediction information of seismic trace. Secondly, to extract 2D-information of data structure by using amplitude difference between predicted seismic trace and original one. Finally, to try to find abnormity regularity of petroliferous data structure by vector association method to achieve the purpose of identification for oil and gas ultimately. This method can be used for identification research on petroliferous layer in exploration well, evaluation well and producing well in different stages from the exploration to development of oil-gas field.

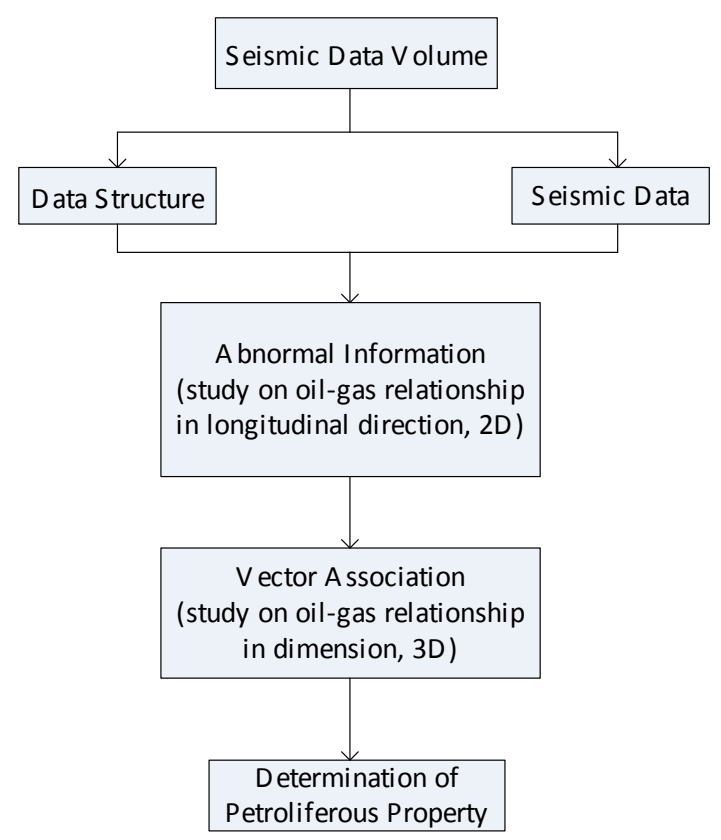

Fig. (1). Thinking for prediction of petroliferous property.

3. SEISMIC PHYSICAL SIMULATION IN SANDSTONE AND MUDSTONE THIN INTERBED UNDER CONDITIONS OF GAS, OIL AND WATER 
In order to study abnormal characteristics of seismic data structure for oil, gas and water, the seismic physical model is established in the laboratory to simulate the process shown in Fig. (2). The total thickness of formation model of sandstone and mudstone thin interbed is $19.77 \mathrm{~mm}$ and the model is composed of plexiglass and sand layer with the thickness $1.22 \mathrm{~mm}$ and $1.43 \mathrm{~mm}$.

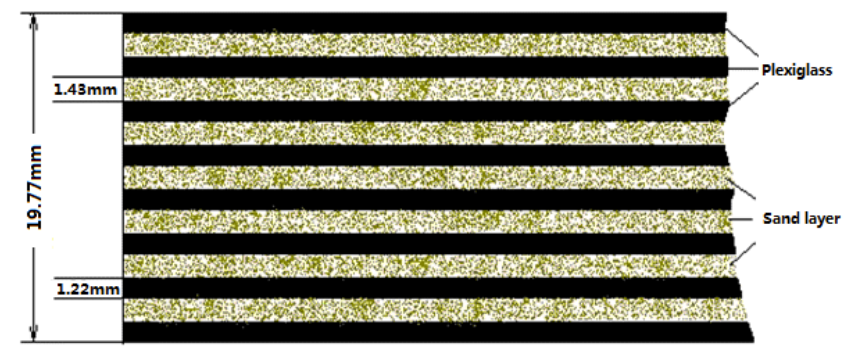

Fig. (2). Modeling diagram of sandstone and mudstone thin interbed.

\section{1) Gas-state simulation}

Physical model with gas-state shows the sand layer contains the air, which is placed in a vacuum incubator. After baking 24 hours with the temperature of $50^{\circ} \mathrm{C}$, the whole model is sealed.

\section{2) Hydrous-state simulation}

Physical model with hydrous-state shows the sand layer contains fully saturated water, which is placed into the water. After stripping seal around, the whole model is immersed in a water container. Then, putting the container into a vacuum tank make thin interbed fill with water by vacuum pumping method. Repeated vacuuming and weighing in order to achieve full saturation state (seven sand pumped into $186 \mathrm{~g}$ of water).

\section{3) Oil-state simulation}

Physical model with oil-state shows the sand layer contains fully saturated oil, which is placed in a vacuum incubator after draining from the sand layer. To make the model bake with the temperature of $50^{\circ} \mathrm{C}$ and vacuumize every 2 hours, re-weighed after 24 hours in order to determine the amount of water discharged. Then, to make the model filled with oil until reaching saturation.

To select appropriate simulation parameters according to actual formation parameters and experimental simulation conditions (as shown in Fig. 3, Fig. 4 and Fig. 5).

\begin{tabular}{ccc}
\hline Parameter & Actual Formation & Physical Model \\
\hline Velocity of P-wave (Vp, 1:1) & $2500 \mathrm{~m} / \mathrm{s}$ & $2500 \mathrm{~m} / \mathrm{s}$ \\
Frequency (f, 1:3000) & $35 \mathrm{~Hz}$ & $105 \mathrm{~Hz}$ \\
Wavelength $(\lambda, 3000: 1)$ & $50 \mathrm{~mm}$ & $1.70 \mathrm{~mm}$ \\
Thin layer thickness (h) & $<5 \mathrm{~m}$ & $1-1.70 \mathrm{~mm}$ \\
$\lambda / \mathrm{h}$ & $>10$ & $>10$ \\
\hline
\end{tabular}

Fig. (3). Model parameter selection.

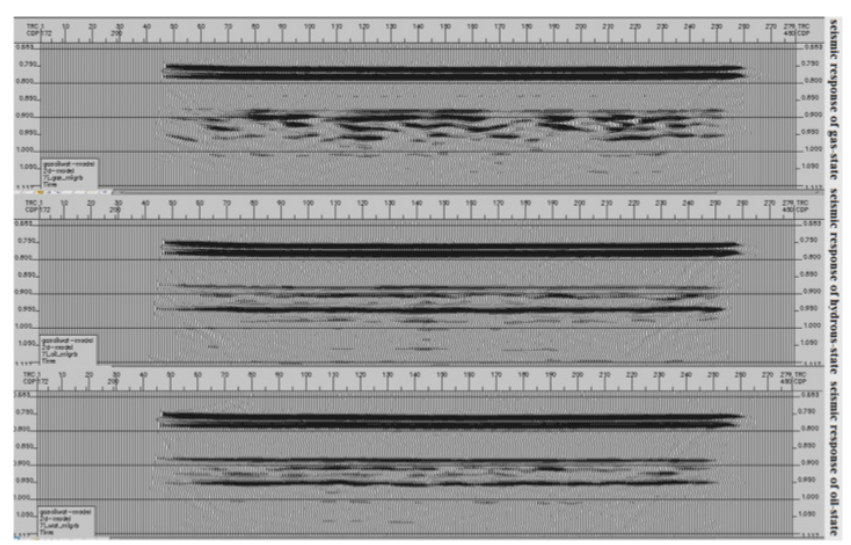

Fig. (4). Seismic records of self-excitation and self-reception under conditions of gas, water and oil.

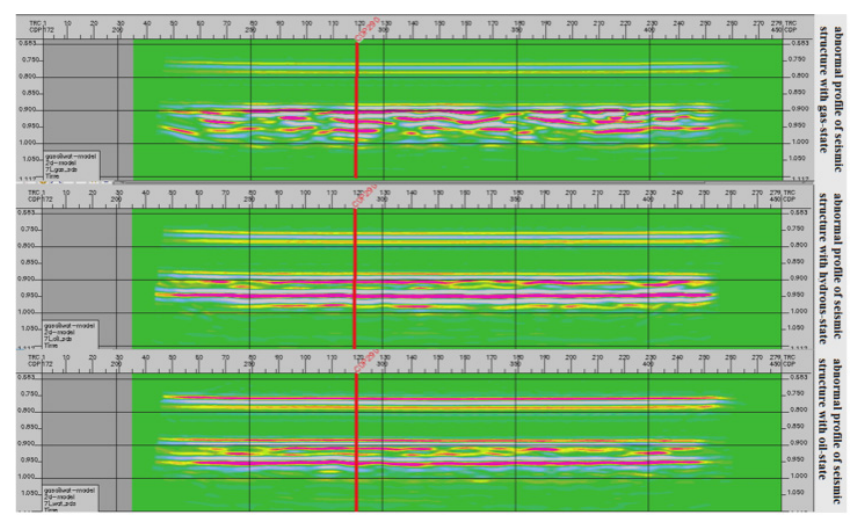

Fig. (5). Abnormal profile map of seismic structure.

To compare the waveform of structure abnormality to the seismic data waveform of CDP (common depth point) at the 290th trace shown in Fig. (6), the red curve stands for structural abnormal one while the black is the curve of seismic records of self-excitation and self-reception, results show the data structure present some kind of abnormal response when the model is under the conditions of gas, water and oil. In the gas-state, structure characteristics change significantly shown as large differences between the amplitude of structure abnormality and original seismic record; in the oil-state, the features are shown as large differences between the amplitude of structure abnormality and original seismic record; in the hydrous-state, the changes of structure characteristics are not obvious shown as small differences between the amplitude of structure abnormality and original seismic record. It can be seen that the seismic data structure can be used to achieve recognition for petroliferous property referred to formations of oil and gas on the basis of obvious abnormal characteristics [9].

\section{APPLICATION AND ANALYSIS RESEARCH}

The target layer of research zone is located in gentle fold belt covering area of $1523 \mathrm{~km}^{2}$, geological overview gives priority to marine deposits, and the main features of distribution include continental shelf facies, platform edge slope facies, open platform facies, platform edge reefs and platform edge shallow facies, reservoirs of reef flat facies are 
developed in good condition. It has completed drilling work of 27 wells (16 wells for testing) in the early. The average altitude of target layer is below $7000 \mathrm{~m}$, seismic SNR (Signal to Noise Ratio) and resolution is relatively low, dominant frequency is generally around $20 \mathrm{~Hz}$. Because of above reasons' influence, it is difficult to conduct horizon comparison and reservoir identification result in the prediction method of conventional petroliferous reservoir is less effective [10] Fig. (7).

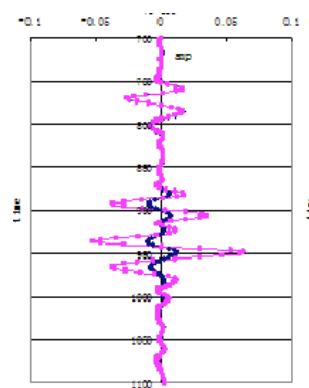

gas-state

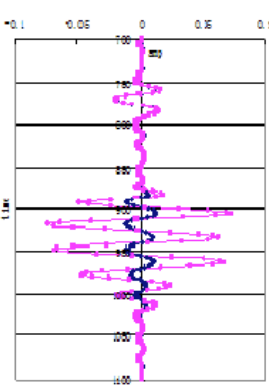

hydrous-state

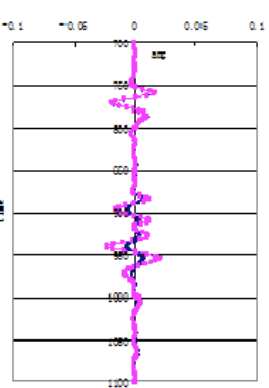

oil-state
Fig. (6). Waveform comparison chart of CDP seismic record of at the 290th trace and data structure under the conditions of gas, water and oil.

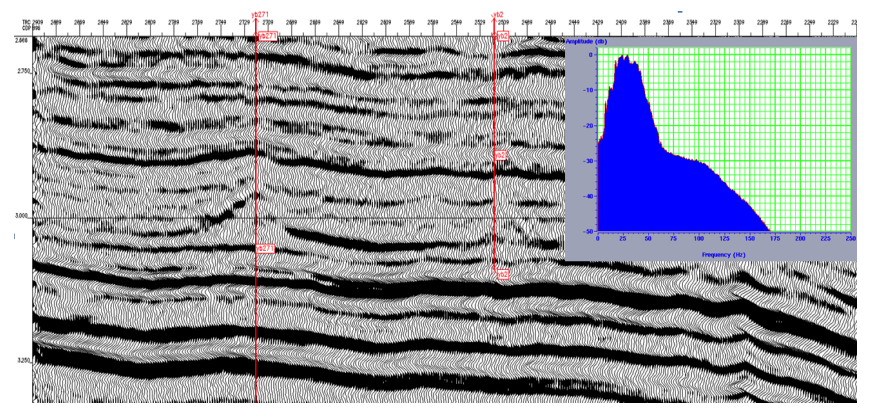

Fig. (7). Seismic profile spectrogram of Inline No. 1945.

According to seismic, logging and testing data of research zone and its sedimentary structure characteristics, using petroliferous prediction method of seismic data structure to conduct research for target layer of the work area. The results show there are obvious abnormities of characteristics of seismic data structure in petroliferous segment (as shown in Fig. 8, Fig. 9).

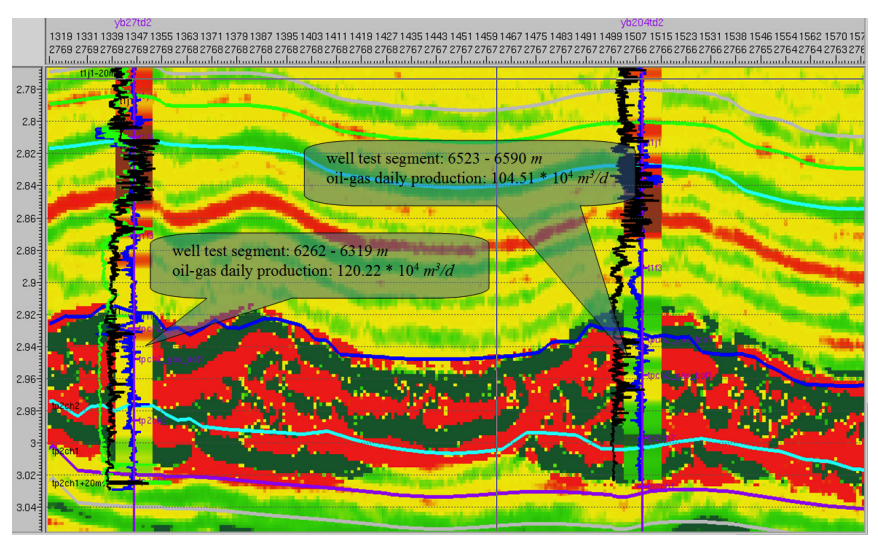

Fig. (8). Seismic data structure profile of yb27- yb204.

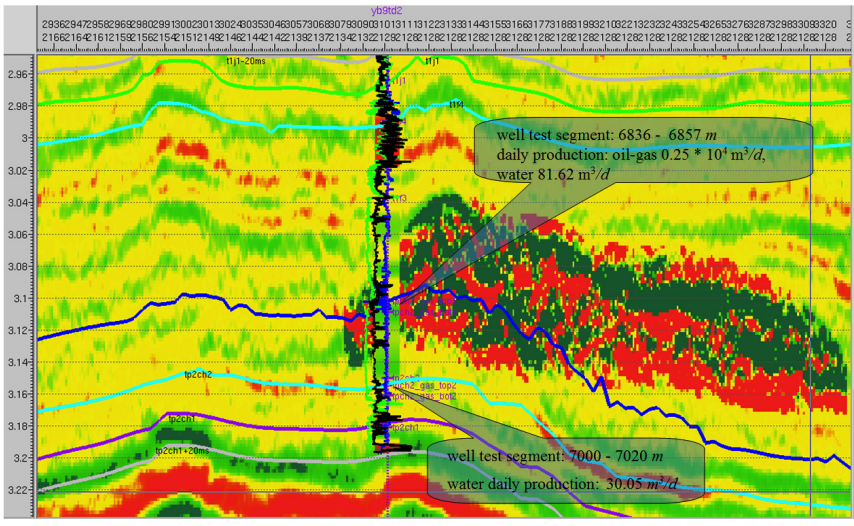

Fig. (9). Seismic data structure profile of yb9.

As shown in Fig. (8) and Fig. (9), the abnormal profile of data structure in the well segment shows in the oil-gas development segment (well yb27 and yb29), the abnormity of structure characteristic is obvious; while in the nondevelopment segment (well yb9), the feature is not obvious. For the above research zone, using abnormal value of seismic data structure for prediction of petroliferous property in target segment, taken top and bottom interface of target segment as time windows to compute RMS (Root-Mean-Square value) of structure characteristic abnormity thereby obtaining its plane distribution map.

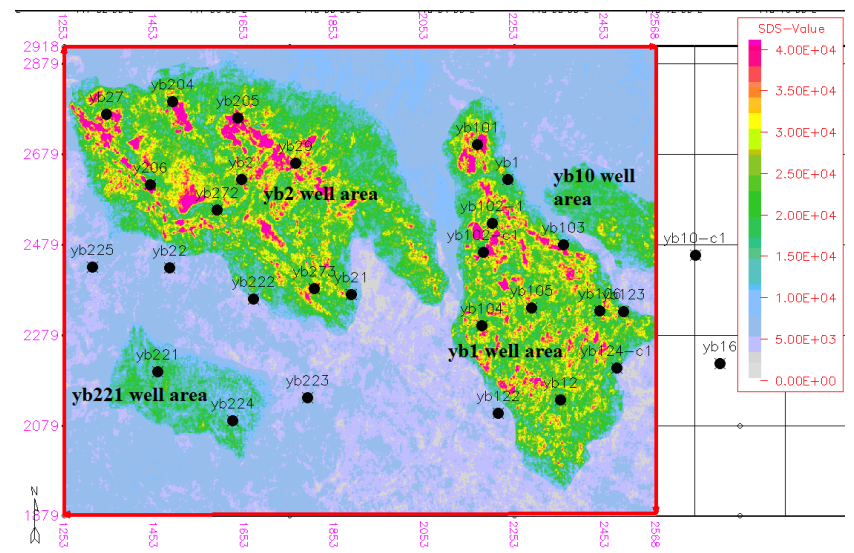

Fig. (10). Plane distribution map of seismic data structure characteristics in target segment.

It can be seen form Fig. (10), there are two main favorable petroliferous unit (yb1 and yb2 well area) and two secondary one (yb10 and yb221 well area) in this research zone. Based on the comparison in prediction and actual test results, it is found that the petroliferous property is preferable in the area where the abnormity of structure characteristic is obvious; when the features are not obvious the petroliferous property is poor. Meanwhile, when the abnormal value is high, the test capacity is higher (well yb27 and yb29); when the abnormal value is low, the test capacity is lower (well yb9). According to the test results from the analysis of known logging data for research zone compared with structure abnormal value (as shown in Fig. 11), it shows: when the structure abnormal value is high (larger than $5.5 * 10^{4}$ ), the target layer usually can be considered as a high-yield formation (larger than $70 * 10^{4} \mathrm{~m}^{3} / \mathrm{d}$ ); when the value is low 
(less than $3.0 * 10^{4}$ ), the target layer usually can be considered as a low-yield formation (less than $10 * 10^{4} \mathrm{~m}^{3} / d$ ); when the value is medium (less than $5.5 * 10^{4}$ and larger than 3.0 $* 10^{4}$ ), the target layer usually can be considered as a medium-yield formation (less than $70 * 10^{4} \mathrm{~m}^{3} / d$ and larger than $10 * 10^{4} \mathrm{~m}^{3} / \mathrm{d}$ ). The statistic results show that the petroliferous property is preferable in the segment where the structure abnormal value is high, while the petroliferous property is poor in the segment where the value is low. That can be used to further proof petroliferous prediction method of seismic data structure is suitable for the zone and the division result of four favorable petroliferous unit is reliable.

\begin{tabular}{|c|c|c|c|c|c|}
\hline $\begin{array}{c}\text { well } \\
\text { number }\end{array}$ & $\begin{array}{c}\text { well test } \\
\text { segment } \\
(m)\end{array}$ & $\begin{array}{l}\text { structure abnormity } \\
\text { characteristics value }\end{array}$ & $\begin{array}{c}\text { oil-gas daily } \\
\text { production } \\
\left(10^{4} m^{3} / d\right)\end{array}$ & $\begin{array}{l}\text { water daily } \\
\text { production } \\
\qquad\left(m^{3} / d\right)\end{array}$ & conclusion \\
\hline yb1c1 & $7331-7368$ & 53779 & 50.31 & non-production & match \\
\hline yb2 & $6545-6593$ & 26262 & 10.24 & 4.51 & match \\
\hline yb2 & $6677-6700$ & 24666 & 4.36 & 16.03 & match \\
\hline$y b 9$ & $6836-6857$ & 27701 & 0.25 & 81.62 & match \\
\hline yb11 & $6797-6917$ & 46655 & 51.63 & non-production & match \\
\hline yb12 & $6692-6780$ & 38483 & 53.14 & non-production & match \\
\hline yb27 & $6262-6319$ & 76347 & 120.22 & non-production & match \\
\hline yb29 & $6808-6820$ & 49229 & 53.52 & non-production & match \\
\hline yb101 & $6955-7022$ & 45081 & 32.13 & non-production & match \\
\hline yb102 & $6711-6791$ & 46528 & 50.55 & non-production & match \\
\hline yb103 & $7047-7695$ & 65574 & 93.91 & non-production & match \\
\hline yb104 & $6700-6726$ & 68069 & 123.32 & non-production & match \\
\hline yb123 & $6904-6918$ & 23956 & 5.32 & 289.91 & match \\
\hline yb124c1 & $6711-6791$ & 31741 & 29.41 & non-production & match \\
\hline yb204 & $6523-6590$ & 76497 & 126.46 & non-production & match \\
\hline yb205 & $6448-6490$ & 67807 & 112.23 & non-production & match \\
\hline yb205 & $6698-6711$ & 63062 & 105.56 & non-production & match \\
\hline
\end{tabular}

Fig. (11). Comparison chart of structure abnormity characteristics and test results.

\section{CONCLUSION}

1) This research indicates seismic data structure reflects the variational regularity of oil-gas-water relationship with a certain degree, which can be used for the identification and classification of petroliferous unit;

2) From plane distribution map of structure abnormity characteristics, the petroliferous property is preferable in the target layer of research zone, yb1 and yb2 well area are main favorable petroliferous unit with structure characteristics are obvious and the distribution range is wide; yb10 and yb221 well area are secondary favorable petroliferous unit with structure characteristics are obvious and the distribution range is wide;
3) In research zone, the distribution of structure abnormality value has closed relationship with target layer capacity, generally speaking, the petroliferous property is preferable when abnormal value is large, while the petroliferous property is poor when the value is small;

4) The plane distribution map of structure abnormity characteristics mainly reflects changes of petroliferous relationship in transverse direction, while the identification and tracking of petroliferous layer still need to use characteristics of structure abnormity profile in longitudinal direction.

\section{CONFLICT OF INTEREST}

The authors confirm that this article content has no conflict of interest.

\section{ACKNOWLEDGEMENTS}

This work is supported by National Natural Science Foundation of China (Grant No. 41474115).

\section{REFERENCES}

[1] C. Lin, L. Sun, and R. Chong, "Numerical Structure of Seismic Data and Hydrocarbon Prediction," China Offshore Oil and Gas (Geology), vol. 6, pp. 417-421, 2000.

[2] J. Deng, "Grey Theory,"Huazhong University of Science and Technology Press, Wuhan, 2003.

[3] C. Lin, S. Wang, Z. Ma, S. Chen, and M. Fang, "Space-time Relationship of Seismic Data Structure and Hydrocarbon Prediction," Petroleum Exploration and Development, vol. 2, pp. 208-215, 2009.

[4] S. Wang, and C. Lin, "The Analysis of Seismic Data Structure and Oil and Gas Prediction," Applied Geophysics, vol. 2, pp. 75-82, 2004.

[5] Z. Yan, Z. Ying, X. Sun, and X. Zheng, "Application of seismic reservoir prediction techniques indifferent exploration and development stages," Petroleum Exploration and Development, vol. 1, pp. 59-63, 2006.

[6] S. Liu, and Z. Xu, "New development of grey system research," Huazhong University of Science and Technology Press, Wuhan, 1996.

[7] M. Ji, S. Wang, S. Li, and S. Chen, "An analysis of attribute prediction result in seismic physical modeling," Petroleum Exploration and Development, vol. 3, pp. 339-341, 2007.

[8] B. He, J. Zhou, and G. Wang, "Using multivariate seismic attributions to predict reservoir information," $O G P$, vol. 3, pp.258-262.

[9] H. Guo, and Q.Liu, "The discussion of earthquake atrribute technology's history, present situation and development tendency," Geophysical \& Geochemical Exploration,vol. 1, pp. 19-22, 2008.

[10] J. Yu, and X. Jiang, "Application of seismic attribute optimization in reservoir prediction," Oil \& Gas Geology, vol. 3, pp.291-295, 2003.

\footnotetext{
Received: May 26, 2015

Revised: July 14, 2015

Accepted: August 10, 2015

(C) Ding et al.; Licensee Bentham Open.
}

This is an open access article licensed under the terms of the (https://creativecommons.org/licenses/by/4.0/legalcode), which permits unrestricted, noncommercial use, distribution and reproduction in any medium, provided the work is properly cited. 